\title{
Atoms and Molecules in a Strong Laser Field
}

\author{
M. Busuladžıćc ${ }^{a, *}$, A. Gazibegović-Busuladžićc ${ }^{b}$, E. Hasović ${ }^{b}$, D.B. Milošević ${ }^{b, c}$ \\ AND W. BECKER ${ }^{c}$ \\ ${ }^{a}$ Medical Faculty, University of Sarajevo, Bosnia and Herzegovina \\ ${ }^{b}$ Faculty of Science, University of Sarajevo, Bosnia and Herzegovina \\ ${ }^{c}$ Max-Born Institut, Berlin, Germany
}

\begin{abstract}
We investigate high-order above-threshold ionization of diatomic molecules and their companion atoms by linearly polarized strong laser field using improved molecular (atomic) strong-field approximation. The most noticeable feature of the molecular spectra is the existence of minima that are absent in atomic case. We have derived an analytical formula for their position which is independent of the molecular symmetry.
\end{abstract}

PACS numbers: $33.80 . \mathrm{Rv}, 32.80 . \mathrm{Rm}, 42.50 . \mathrm{Hz}$

\section{Introduction}

Atomic and molecular processes in intense laser fields have received much attention in the last decade $[1,2]$. Irradiating atomic or molecular targets by an intense laser field can lead to a few highly nonlinear phenomena. In particular, two of them, high-order above-threshold ionization (HATI) and high-order harmonic generation (HHG) are of great interest for deeper understanding of the laser-matter interaction. The physics of these processes is usually described using the three-step model. The first step is the same for both of the above-mentioned processes. Atom or molecule is ionized by absorption of more photons than is necessary for ionization: this process is called above-threshold ionization (ATI). If the ionized electron goes directly to the detector we call this process direct ATI. Also, it is possible that the electron driven by the laser field revisits its parent atomic (molecular) ion. The returning electron can recombine with parent ion emitting a high-order harmonic: this is the HHG process. It may also elastically scatter off its parent ion and then leave it towards the detector. In the last case the electron can absorb many more photons from the laser field than in the direct ATI. This process was named high-order ATI (HATI). In this paper we are interested in HATI process on molecules and its companion atoms, i.e. atoms which have almost the same ionization potential as considered molecules.

\section{General theory}

In order to describe exactly behavior of atom or molecule in a strong laser field one should solve the corresponding time-dependent Schrödinger equation. Unfortunately, it is very difficult to carry out such calculations

\footnotetext{
* corresponding author; e-mail: mustafabusuladzic@bih.net.ba
}

for more complex atoms, and specially for molecular targets. In these circumstances physicists have relied on some kind of approximation. In Ref. [3] we have developed improved strong-field approximation (SFA) in order to simulate the HATI experiments with noble gases. The SFA approach does not account for the effects of excited bound states, nor does it contain the consequences of the final-state interaction of the freed electron with its parent ion except, in its generalized form, for one "hard" interaction upon a recollision. Interaction between ionized electron and laser field can be expressed in different gauges. In the atomic case we used the standard length gauge. It was shown that, within the SFA, for such choice of gauge the ATI spectra of negative ions are in accordance with those obtained by solving the time-dependent Schrödinger equation [4].

In Ref. [5] we have considered a diatomic molecule as a three-particle system consisting of two heavy atomic (ionic) centers and an electron and have introduced our modified molecular SFA theory of ATI. After separation of the center-of-mass coordinate, the dynamics of this system is reduced to the relative electronic and nuclear coordinates. We introduced two forms of molecular SFA, one with the field-free and other with the field-dressed initial molecular bound states. We present here the final result for the dressed modified molecular SFA in length gauge for neutral homonuclear diatomic molecules. The rate of the ATI process with absorption of $n$ photons from the laser field is equal to $2 \pi p_{\mathrm{f}}\left|T_{\mathrm{f}}(n)\right|^{2}$, where the $T$-matrix element $T_{\mathrm{fi}}(n)$ is the Fourier transform of an expression which is proportional to [5]:

$$
\begin{aligned}
& \mathcal{T}_{\mathrm{fi}}^{(0) d}(t) \\
& =\sum_{s} \mathrm{e}^{\mathrm{i} s} \boldsymbol{p}_{\mathrm{f}} \cdot \boldsymbol{R}_{0} / 2\left\langle\boldsymbol{p}_{\mathrm{f}}+\boldsymbol{A}(t)|\boldsymbol{r} \cdot \boldsymbol{E}(t)| \Psi_{s}^{(0)}\right\rangle .
\end{aligned}
$$

In the last equation $\boldsymbol{p}_{\mathrm{f}}$ is the final momentum of detected electron, $\boldsymbol{R}_{0}$ is the internuclear axis, while $\boldsymbol{r} \cdot \boldsymbol{E}(t)$ 
is the interaction with the laser field in length gauge and dipole approximation (atomic system of units is used). The ground-state molecular electronic wave function is defined as linear combination of atomic orbitals, i.e. $\left|\Psi_{s}^{(0)}\right\rangle=\sum_{a} c_{s a}\left|\psi_{a}^{(0)}\right\rangle$. In our notation, the connection between the coefficients $c_{s a}$ depends on the symmetry of the considered molecule and is given by $c_{-1 a}=s_{a \lambda} c_{1 a}$, with

$$
\begin{aligned}
& s_{a \lambda}=(-1)^{l_{a}-m_{a}}(-1)^{m_{\lambda}}, \\
& s_{a \lambda}=(-1)^{l_{a}-m_{a}}(-1)^{m_{\lambda}+1},
\end{aligned}
$$

for $\mathrm{u}$ and $\mathrm{g}$ symmetry, respectively. Here, $m_{\lambda}$ is the projection of the orbital angular momentum on the internuclear axis. For example, for $\sigma$ states it is $m_{\lambda}=0$, while for $\pi$ states it is $m_{\lambda}=1$. The factor $(-1)^{l_{a}-m_{a}}$ comes from the inversion of the $z$ coordinate of the second center.

In the subsequent papers [6-8], we have generalized this theory to include the rescattering of the ionized electron off the parent molecular ion.

Following mentioned procedure we got two terms, first of them describes the direct ATI, and it was given by Eq. (1). The second term that corresponds to the rescattered electrons is

$$
\begin{aligned}
& \mathcal{T}_{\mathrm{fi}}^{(1) d}(t)=-\mathrm{i}^{-\mathrm{i} S_{k_{s t}}(t)} \int_{0}^{\infty} \mathrm{d} \tau\left(\frac{2 \pi}{\mathrm{i} \tau}\right)^{3 / 2} \\
& \quad \times \exp \left(\mathrm{i}\left[S_{\boldsymbol{k}_{s t}}\left(\tau^{\prime}\right)-\Delta E\left(\boldsymbol{R}_{0}\right) \tau\right]\right) \exp \left(-\frac{\mathrm{i}}{2 \tau} \frac{\partial^{2}}{\partial \boldsymbol{k}^{2}}\right) \\
& \quad \times \sum_{s^{\prime}} V_{e}^{s^{\prime}} \boldsymbol{K} \sum_{s} \exp \left(\left(s k-s^{\prime} \boldsymbol{K}\right) \cdot \boldsymbol{R}_{0} / 2\right) \\
& \quad \times\left\langle\boldsymbol{k}+\boldsymbol{A}\left(\tau^{\prime}\right)\left|\boldsymbol{r} \cdot \boldsymbol{E}\left(\tau^{\prime}\right)\right| \Psi_{s}^{(0)}\right\rangle_{\mid k=k_{s t}}
\end{aligned}
$$

where $\boldsymbol{k}_{s t}$ is the stationary intermediate electron momentum between the ionization and rescattering (it is in the direction of the laser electric field vector), while $S_{\boldsymbol{k}}$ is action defined by $S_{\boldsymbol{k}}(t)=\int^{t} \mathrm{~d} t^{\prime}\left[\boldsymbol{k}+\boldsymbol{A}\left(t^{\prime}\right)\right]^{2} / 2$.

$V_{e K}^{s^{\prime}}$ is the Fourier transform of the rescattering potential at the different atomic (ionic) centers, where $\boldsymbol{K}=\boldsymbol{k}-\boldsymbol{p}_{\mathrm{f}}$. The rescattering potential was modeled by a static double Yukawa potential as in Ref. [3].

As molecules are multicentre systems, the ionization as well as rescattering can happen at different centers, causing interference structures in the electron spectrum. So, our matrix element related to rescattering process can be decomposed into four terms

$$
\begin{aligned}
& T^{(1)}=T^{++--}+T^{+-}+T^{-+}, \\
& T^{++--} \equiv T^{++}+T^{--} .
\end{aligned}
$$

The electron can tunnel out of either atom. These possibilities are denoted by superscript "+" or "-". With $T^{++}\left(T^{--}\right)$we denote the $T$-matrix contribution of the electron which, after excursion in the continuum, rescatters at the same center "+" ("-"). The terms $T^{+-}$ and $T^{-+}$correspond to the cases where the electron is ionized and rescatters off different centers. It can be shown that our matrix element is proportional to $\cos \left(\left(\boldsymbol{p}_{\mathrm{f}}-\boldsymbol{k}_{s t}\right) \cdot \boldsymbol{R}_{0} / 2\right)$, regardless of the symmetry of the atomic orbital of which the considered molecular orbital consists. Therefore, if we present the rescattering ionization rate in the $\left(E_{p_{\mathrm{f}}}, \theta\right)$ plane, we will have local minima if the condition

$$
R_{0}\left|p_{\mathrm{f}} \cos \theta-k_{s t} \cos \theta_{\mathrm{L}}\right|=(2 m+1) \pi,
$$

with $m$ integer, is fulfilled. For the laser and molecular parameters we are considering in the present paper only the lowest value $m=0$ contributes. By $\theta_{\mathrm{L}}$ we define angle between the laser polarization vector and internuclear axis, while $\theta$ accounts for the angle between final momentum of the detected electron and internuclear axis. This novel two-source two-rescattering-centers interference has been observed in experiments with unaligned molecules $[9,10]$. Recently, we have shown that for elliptical polarization interference condition (5) still holds [11] but one should have in mind that $\boldsymbol{k}_{s t}$ is a complex solution of the saddle-point equations $[12,13]$.

\section{Numerical results}

We will consider $\mathrm{N}_{2}$ and $\mathrm{O}_{2}$ molecules as well as their companion atoms, $\mathrm{Ar}$ and $\mathrm{Xe}$, respectively. For the $\mathrm{N}_{2}$ molecule, the initial HOMO is $3 \sigma_{\mathrm{g}}$. Twelve atomic orbitals with $m_{a}=0$ are taken into account [5-7]. The HOMO of the $\mathrm{O}_{2}$ molecule is $1 \pi_{\mathrm{g}}$ and we chose five atomic orbitals having $m_{a}=1$. For $\mathrm{N}_{2}\left(\mathrm{O}_{2}\right)$ the equilibrium internuclear distance is $R_{0}=2.068$ a.u. (2.282 a.u.) and its ionization energy is $I_{p}=15.58 \mathrm{eV}(12.03 \mathrm{eV})$. In the case of Ar we have $I_{p}=15.76 \mathrm{eV}$, while for Xe the ionization potential is $I_{p}=12.13 \mathrm{eV}$. The laser field is linearly polarized with wavelength $800 \mathrm{~nm}$ and intensity $I=1.68 \times 10^{14} \mathrm{~W} / \mathrm{cm}^{2}$. For both molecules we choose $\theta_{\mathrm{L}}=75^{\circ}$.

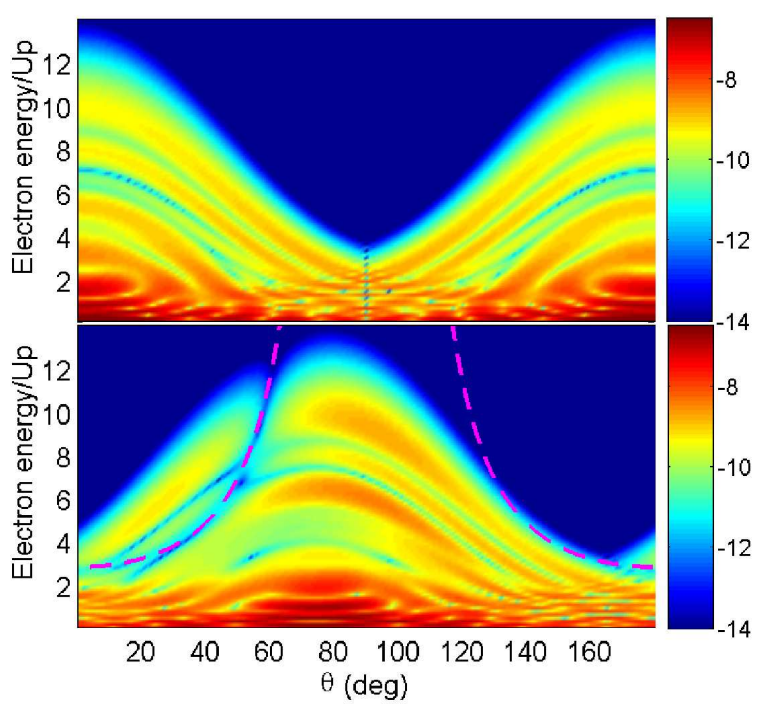

Fig. 1. Logarithm of the differential ionization rate of Ar (upper part) and $\mathrm{N}_{2}$ (bottom part), coded in false colors in the $\left(E_{p_{f}}, \theta\right)$ plane. The intensity of the linearly polarized laser field is $I=1.68 \times 10^{14} \mathrm{~W} / \mathrm{cm}^{2}$, and the wavelength is $800 \mathrm{~nm}$. In the bottom panel we have $\theta_{\mathrm{L}}=75^{\circ}$. The dashed curve is based on Eq. (6) (see the text). 


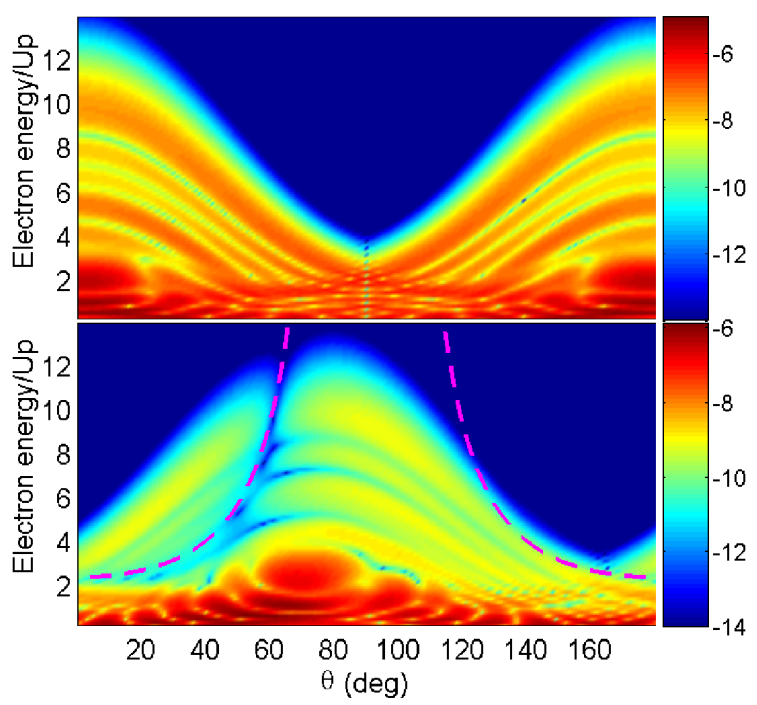

Fig. 2. The same as in Fig. 1 but for Xe (upper part) and $\mathrm{O}_{2}$ (bottom part).

In Figs. 1 and 2, we compare angle-resolved ATI spectra for $\mathrm{Ar}$ and for $\mathrm{N}_{2}$, and for $\mathrm{Xe}$ and for $\mathrm{O}_{2}$, respectively. The most important difference between the atomic and molecular spectra is the existence of minima in molecular spectra, particularly in the cutoff region, that are absent in the atomic case. These minima are determined by the condition given by Eq. (5) for $m=0$ which leads to

$$
\left(p_{\mathrm{f}} \cos \theta-k_{s t} \cos \theta_{\mathrm{L}}\right)^{2}=\pi^{2} / R_{0}^{2} \text {. }
$$

According to the quantum-orbit theory [12, 13], the shortest electron orbits correspond to ionization at the time near an extremum of the electric field and rescattering at the time near the second-to-next zero crossing. In our case, for $A(t)=A_{0} \cos \omega t, k_{s t} \approx-2 A_{0} /(3 \pi)$. This yields an approximation to $(6)$, represented by the dashed pink lines in bottom parts in both figures. We have also found that, for $\theta_{\mathrm{L}}=0^{\circ}$ and $\theta_{\mathrm{L}}=90^{\circ}$, the behavior of the $\mathrm{O}_{2}\left(\pi_{\mathrm{g}}\right)$ molecule is dramatically different from that of the $\mathrm{N}_{2}\left(\sigma_{\mathrm{g}}\right)$ molecule. In fact, for $\mathrm{O}_{2}$ and the above-mentioned two specific molecular orientations, the high-energy plateau is entirely absent. For parallel orientation this was explained by the fact that the rescattering matrix element is proportional to $\sin \theta_{\mathrm{L}}=0$. For perpendicular orientation it was shown analytically [6] that $T \propto 1+s_{a \lambda}$. Therefore, partial contributions of $p$ orbitals, for which $s_{a \lambda}=-1$, vanish. In addition, the partial contribution of the even $d$ orbitals $\left(s_{a \lambda}=1\right)$ is proportional to $\cos \theta_{\mathrm{L}}$, so that it also vanishes for $\theta_{\mathrm{L}}=90^{\circ}$. In contrast, for $\mathrm{N}_{2}$ we have a substantial yield of this part of the spectrum in both cases.

\section{Conclusions}

We have considered atomic and molecular HATI spectra generated by a linearly polarized laser field. In the case of diatomic molecules there are four possible contributions to the HATI spectrum due to its two-centers structure. As a consequence one can observe minima in the molecular spectra which are absent in atomic case. Dramatically different behavior was found between $\mathrm{N}_{2}$ $\left(\sigma_{\mathrm{g}}\right)$ and $\mathrm{O}_{2}\left(\pi_{\mathrm{g}}\right)$ at parallel and perpendicular molecular orientation. This is crucial for explanation of the two-source double-slit destructive interference effect, which has been observed in experiments with randomly oriented $\mathrm{O}_{2}$ molecules [10].

\section{Acknowledgments}

This work was supported by the Federal Ministry of Education and Science, Bosnia and Herzegovina.

\section{References}

[1] W. Becker F. Grasbon, R. Kopold, D.B. Milošević, G.G. Paulus, H. Walther, Adv. At., Mol., Opt. Phys. 48, 35 (2002).

[2] M. Lein, J. Phys. B 40, R135 (2007).

[3] E. Hasović, M. Busuladžić, A. GazibegovićBusuladžić, D.B. Milošević, W. Becker, Laser Phys. 17, 376 (2007).

[4] D. Bauer, D.B. Milošević, W. Becker, Phys. Rev. A 72, 023415 (2005).

[5] D.B. Milošević, Phys. Rev. A 74, 063404 (2006).

[6] M. Busuladžić, A. Gazibegović-Busuladžić, D.B. Milošević, W. Becker, Phys. Rev. Lett. 100, 203003 (2008).

[7] M. Busuladžić, A. Gazibegović-Busuladžić, D.B. Milošević, W. Becker, Phys. Rev. A $\mathbf{7 8}$, 033412 (2008).

[8] D.B. Milošević, E. Hasović, S. Odžak, M. Busuladžić, A. Gazibegović-Busuladžić, W. Becker, Laser Phys. 19, 185 (2009).

[9] M. Okunishi, R. Itaya, K. Shimada, G. Prümper, K. Ueda, M. Busuladžić, A. Gazibegović-Busuladžić, D.B. Milošević, W. Becker, J. Phys. B 41, 201004(F) (2008).

[10] M. Okunishi, R. Itaya, K. Shimada, G. Prümper, K. Ueda, M. Busuladžić, A. Gazibegović-Busuladžić, D.B. Milošević, W. Becker, Phys. Rev. Lett. 103 , 043001 (2009).

[11] M. Busuladžić, A. Gazibegović-Busuladžić, D.B. Milošević, Phys. Rev. A 80, 013420 (2009).

[12] R. Kopold, D.B. Milošević, W. Becker, Phys. Rev. Lett. 84, 3831 (2000).

[13] P. Salières, B. Carré, L. Le Déroff, F. Grasbon, G.G. Paulus, H. Walther, R. Kopold, W. Becker, D.B. Milošević, A. Sanpera, M. Lewenstein, Science 292, 902 (2001). 